\title{
MEMORIAL DA TERRA: AMZ.21
}

Memorial From Earth: AMZ.21

Memorial de la Tierra: AMZ.21

$>$ Hugo Nascimento [Museu Paraense Emilio Goeldi, Brasil]*

Citação recomendada: NASCIMENTO, Hugo Memorial da Terra AMZ.21. Revista Poiésis, Niterói, v. 22, n. 38, p. $21-43$, jul./dez. 2021. [https://doi. org/10.22409/poiesis.v22i38.45678] Este documento é distribuído nos termos da licença Creative Commons Atribuição-NãoComer cial 4.0 Internacional [CC-BY-NC] (C) 2021 Hugo Nascimento

(Submetido: 31/8/2020; Aceito: 7/1/2021; Publicado: 7/7/2021)
MEMORIAL DA TERRA é um projeto de investigação permanente que busca propor desde os lugares da arte, miradas críticas acerca das dinâmicas territoriais na Amazônia - os modelos de ocupação e exploração, as violências estruturais, os modos de representação, os processos de identificação, desvios e silenciamentos. Trabalho de inventariação sempre inacabado, pode assumir diferentes formas ao se tornar público. Desde os arquivos acumulados, AMZ.21 apresenta um remix de fotocópias produzidas através de emulsões fotosensíveis de jenipapo e urucum, extraídas e processadas na llha de Caratateua, com nomes de 420 pessoas, ambientalistas, indígenas, sindicalistas, quilombolas, trabalhadores sem-terra, vítimas diretas dos conflitos pela terra na Amazônia brasileira do século XXI .

> Belém, Amazônia, 1988. Hugo Nascimento é artista visual, músico e arte-educador. Iniciou suas pesquisas em fotografia em 2007. Em 2009, ingressou no curso de Artes Visuais [UFPA]. Integrou projetos de pesquisa [Per- cursos da Imagem na Arte Contemporânea - CNPQ] e extensão [Territórios Híbridos - PIBEX]. Participou de diversas mostras coletivas [Sesc Universitário, 2009-10, Primeiros Passos, 2011 - 12, Xumucuis, 2013-14] e residências artísticas [LABCLUBE-RJ, 2015, Lastro y Materia Gris, BOL, 2017, CorpusUrbi-AP, 2018, CopperLeg Residence, EST. 2019, Rucka, LET. 2019]. Também possui pesquisas em vídeo-ativismo [como Amazônia em Chamas], cartografias críticas e intervencionismo urbano [LABCART], e em cultura popular [Carimbó Cobra Venenosa, Tamuatás do Tucunduba]. Atualmente é discente do programa de pós-graduação em Diversidade Sóciocultural do Museu Paraense Emílio Goeldi cuja pesquisa busca desenvolver um mapeamento das relações arte território na Amazônia do século XXI. <

\footnotetext{
* E-mail: hugartss@gmail.com, ORCID: https://orcid.org/0000-0001-6414-5768
} 
[...] Ivo Azevedo dos Santos, Ribeirinho, Coari-AM, 11/1/2000 / Trabalhador rural desconhecido, Santana do Araguaia-PA, 31/5/2000 / José Dutra da Costa [Dezinho], Dirigente Sindical, Rondon do Pará, 21/11/2000 / José Ribamar de Souza, Sem-terra, São João do Araguaia, 13/2/2000 / Neuci Barbosa da Silva, Liderança, Parauapebas, 30/5/2000 / João Batista Pereira, Assentado, Curionópolis, 7/5/2000 / Osvaldo Bonifácio dos Santos, Assentado, Araguacema-TO, 21/5/2000 / Francisco de Souza Silva, Sitiante, Porto Velho-RO, 4/8/2000 / José de Jesus Santana, Posseiro, Chapadi- nha-MA, 2/5/2001 / Eliézer Cândido Costa, Assentado, Pio XII-MA, 6/5/2001 / Antônio Firme da Silva, Assentado, São João do Araguaia-PA, 20/4/2001 / Cleonice Campos Lima, Liderança, Marabá-PA, 9/7/2001 / Francisco Xavier Ferreira Nunes, Sem-terra, Itupiranga- PA, 9/7/2001 / José Pinheiro Lima, Liderança, Marabá-PA, 9/7/2001 / Samuel Campos Lima, Sem-terra, Marabá-PA, 9/7/2001 / Ademir Alfeu Federicci [Dema], Dirigente sindical, Anapu-PA, 25/8/2001 / Miguel Freitas da Silva, Liderança, Baião-PA, 1/92001 / Gilson Souza Lima, Liderança, Marabá/Parauapebas-PA, 5/10/2001 / Maria de Nazaré Moraes Maués, Posseiro, Abaetetuba-PA, 9/7/2002 / Geraldo, Posseiro, Abel Figueiredo- PA, 1/3/2002 / Osvaldino Viana de Almeida, Posseiro, Afuá-PA, 20/10/2002 / Bartolomeu Morais da Silva, "Brasília", Ribeirinho, Altamira/Novo Progresso-PA, 21/7/2002 / Mineirinho, Dirigente sindical, Baião-PA, 20/10/2002 / Euzival Nascimento Maranhão, Sem-terra, Bannach Pista Branca-PA, 1/11/2002 / Semir Magalhães, Assentado, Cumaru do Norte-PA, 7/10/2002 / Antônio Clênio Cunha Lemos, Assentado, Curionópolis/Parauapebas-PA, 17/11/2002 / Ivo Lau- rindo do Carmo, Dirigente sindical, Irituia Km 19 da Belém Brasília, 27/6/2002 / Wilson Cardec Bento dos Santos, Li- derança Marabá-PA, 24/8/2002 / Raimundo Alves, Sem-terra, Novo Repartimento-PA, 25/12/2002 / Valdir Sabino da Silva, Assentado, Novo Repartimento-PA, 6/11/2002 / Valdivan Ferreira da Silva, Trab. Rural, Novo Repartimento-PA, 6/11/2002 / Magno Fernandes do Nascimento, Trab. Rural, Rondon do Pará, 10/9/2002 / Amarair Câmara da Silva, Posseiro, São Félix do Xingu-PA, 13/6/2002 / Edvan Pereira, Sem-terra, Xinguara-PA, 1/6/2002 / Deuzimar, Trabalha- dor Rural, Xinguara/Rio Maria-PA, 23/2/2002 / Nivaldo, sobrinho de Raimundo, Assentado, Xinguara/Rio Maria-PA, 23/2/2002 / Olavo, Filho de Deuzimar, Assentado, Xinguara/Rio Maria-PA, 23/2/2002 / Raimundo, Assentado, Xingua- ra/Rio Maria-PA, 23/2/2002 / Raimundo Ferreira de Oliveira, Trab. Rural, Buritis-PA, 13/7/2002 / Ivo Martins dos Santos, Posseiro, Nova Mamoré-RO, 30/7/2002 / Ozéias Martins de Souza, Assentado, Jacinópolis-GO 27/11/2002 / Regis, Assentado, Buriticupu-MA, 4/4/2003 / Raimundo de Aquino Filho, Posseiro, Bacabal-MA, 9/7/2003 / Antônio Gregório da Conceição, Assentado, Bacabal-MA,

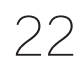





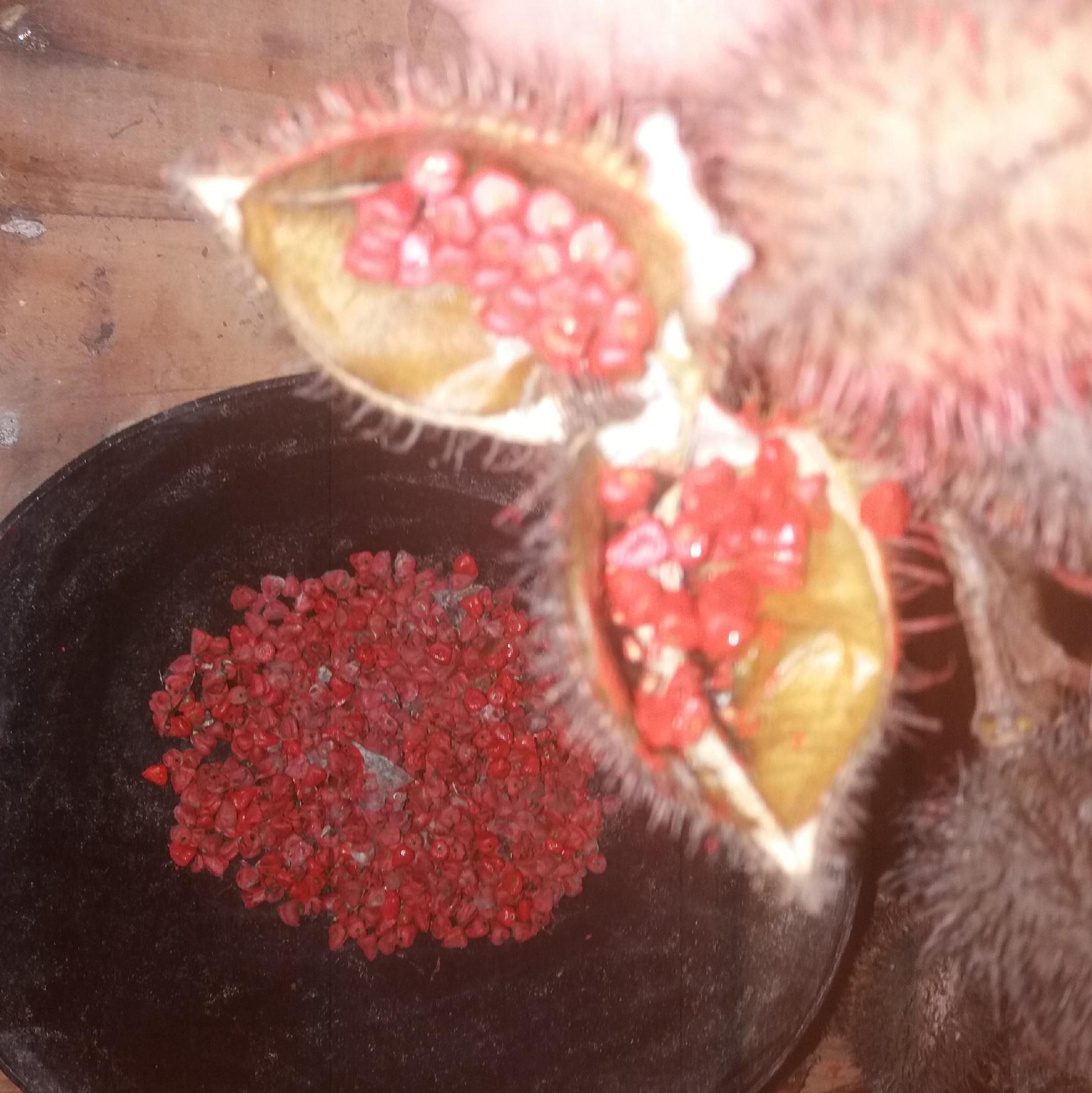



9/7/2003 / João Maria Chaves, Trab. Rural, Colniza-MT, 10/11/2003/ José Carlos Chaves, Assentado, Colniza-MT, 10/11/2003 / Milton, Assentado, Vila Rica-MT, 3/11/2003 / Urana, As- sentado, Vila Rica-MT, 3/11/2003 / Antônio Vasquez Cardoso, Ribeirinho, Afuá-PA, 16/2/2003 / Raimundo Braga, Ribeirinho, Afuá-PA, 8/3/2003 / Antônio Henrique Martins, Liderança, Altamira-PA, 22/2/2003 / Cícero Ferreira Lima, Sem-terra, Altamira-PA, 2/6/2003 / Raimundo Guilherme da Silva, Sem-terra, Baião-PA, 6/5/2003 / Joaquim de Jesus Figueiredo, Sem-terra, Baião-PA, 27/9/2003 / Denerval Vieira dos Santos, Agrimensor, Cumaru do Norte-PA, 3/1/2003 / Raimundo Félix dos Santos, Criança, Cumaru do Norte, 3/1/2003 / José Mendes, Dirigente sindical, Curio- nópolis/Paraupebas Garimpo de Serra Pelada-PA, 22/1/2003 / Raimundo Amorim, Garimpeiro, Curionópolis/Paraupe- bas Garimpo de Serra Pelada-PA, 25/1/2003 / José do Carmo Silva "Dodô", Assentado, Marabá-PA, 7/3/2003 / Osval- do Pereira dos Santos, Assentado, Marabá-PA, 14/4/2003 / Assentado do Fronteiras Aparecido "Cido", Assentado, Novo Progresso-PA, 27/7/2003 / Luiz Carlos Campos "Negão", Liderança, Novo Repartimento-PA, 27/1/2003 / Solam, Trab. Rural, Novo Repartimento-PA, 30/5/2003 / Francisco Bento da Silva, Posseiro, Novo Repartimento-PA, 25/8/2003/ Trabalhador rural da Gleba Capivara, Trab. Rural, Novo Repartimento-PA, 24/9/2003 / Posseiro da Gleba Belo Monte [filho], Posseiro, Novo Repartimento/Anapu-PA, 31/3/2003 / Posseiro da Gleba Belo Monte [pai], Trab. Rural, Novo Repartimento/Anapu-PA, 31/3/2003 / Posseiro da Gleba Belo Monte[genro], Posseiro, Novo Repartimento/ Anapu-PA, 31/3/2003 / Juarez Pereira da Conceição, Sem-terra, Piçarra-PA, 17/4/2003 / Rosildo Lima, Trab. Rural, Santana do Araguaia-PA, 6/4/2003 / José Orlando de Souza, Liderança, Santarém-PA, 3/5/2003 / Justino Pereira da Silva, Trab. Rural, São Félix do Xingu-PA, 12/9/2003 / Pedro Formiga, Trab. Rural, São Félix do Xingu-PA, 12/9/2003 / Penteado, Trab. Rural, São Félix do Xingu-PA, 12/9/2003 / Antônio Vieira da Silva, São Félix do Xingu-PA, 12/9/2003 / Antônio da Conceição, Trab. Rural, São Félix do Xingu-PA, 12/9/2003 / Eliseu, Trab. Rural, São Félix do Xingu-PA, 12/9/2003/ Maurício, Trab. Rural, São Félix do Xingu-PA, 12/9/2003 / Baixinho Moreno, Trab. Rural, São Félix do Xingu-PA, 12/9/2003 / José "Ceará", Trab. Rural, São Félix do Xingu-PA, 29/6/2003 / Mauro Xavier dos Santos, Sem-terra, São Félix do Xingu-PA, 1/7/2003 / Serafim José de Santana, Liderança, Ariquemes-PA, 1/8/2003 / Antônia Santana de Cas- tro, Liderança, Ariquemes-RO, 1/8/2003 / Edgar Trevisan, Liderança, Cujubim-RO, 20/7/2003 / Devair Cordeiro Ver- bano, Posseiro, Nova Mamoré-RO, 6/10/2003 / Osvaldo Pereira, Posseiro, Nova Mamoré-RO, 6/10/2003 / Evaldo Hil- ton Margoto Verbano, Posseiro, Nova Mamoré-RO, 6/10/2003 / João Olegário da Silva, Posseiro, Nova

\section{6}


Mamoré-RO, 6/10/2003 / Benedito, Posseiro, Porto Velho-RO, 5/2/2003 / Aldo da Silva Mota, Índio, Uiramutã Raposa Serra do Sol e Surumu-RR, 9/1/2003 / Antônio Fernandes de Araújo, Assentado, Natividade-RR, 26/8/2003 / Vitória Filha do Nas- cimento, Assentada, Natividade, 26/8/2003 / José Borges da Silva, Posseiro, Aldeias Altas Povoado Jaburu-MA, 21/01/2004 / Evaldo, Posseiro, Nina Rodrigues-MA, 06/02/2004/José Wires, Sem-terra, Anapú PDS Esperança-PA, 29/05/2004 / Jerônimo Gonçalves Souza, Assentado, Cumaru do Norte, 30/10/2004 / Manoel Pereira, Assentado, Cuma- ru do Norte-PA, 30/10/2004 / Adilson Prestes, Ag. pastoral, Novo Progresso-PA, 03/07/2004 / Gil, Trab. Rural, Novo Repartimento-PA, 20/01/2004 / Eudes, Trab. Rural, Novo Repartimento-PA, 20/01/2004 / José Antônio Pereira de Sou- za, Assentado, Novo Repartimento-PA, 30/03/2004 / Gaspar, Trab. Rural, Novo Repartimento-PA, 30/05/2004 / Antô- nio, Trab. Rural, Novo Repartimento-PA, 19/08/2004 / José Ribamar Ribeiro, Assentado, Pacajá-PA, 28/02/2004 / Ri- bamar Francisco dos Santos Presidente de STR, Rondon do Pará-PA, 06/02/2004 / Antônio "da farinha", Santa Maria das Barreiras-PA, 01/02/2004 / Ezequiel de Moraes Nascimento, Liderança, Santa Maria das Barreiras-PA, 29/01/2004/ Epitácio Gomes da Silva, Tailândia-PA, 23/03/2004 / Manoel da Silva Costa, Trab. Rural, Tailândia-PA, 03/05/2004 /Valdez Marinho Lima, Índio, Mucajaí Área Indígena lanomâmi-RR, 23/02/2004 / Eustáquio Carvalho, Posseiro, Caja- pió-MA, 02/08/2005 / Araújo Guajajara, ìndio, Grajaú Bacurizinho/Aldeia Kamihaw Guajajara/Nazaré-MA, 21/05/2005/Jerônimo, Sem-Terra, Peixoto de Azevedo/ Nova Guarita-MT, 09/03/2005 / Mauro Gomes Duarte, Sem-terra, Peixoto de Azevedo/Nova Guarita-MT, 16/11/2005 / Vanderlei Macenas Cruz, Sem-terra, Peixoto de Azevedo/Nova Guarita-MT, 16/11/2005 / Dorothy Mae Stang, Religiosa, Anapu Gleba Bacajá/PDS Esperança-PA, 12/02/2005 / Raimundo Nonato Pereira de Moraes, Liderança, Anapu Gleba Belo Monte/PDS Virola Jatobá-PA, 13/5/2005 / Claúdio Dantas Muniz, Assentado, Anapu Gleba Manduacari-PA, 15/2/2005/ José Pereira da Silva, Sem-terra, Canaã dos Carajás-PA 15/6/2005 / Ivandro Rodrigues, Presidente de STR, Castanhal-PA, 9/1/2005 / Domingos Farias dos Santos, Sindicalista, Itupiranga, 8/11/2005 / Rony Silva da Conceição, Assentado, Marabá-PA, 19/2/2005 / Pedro Laurindo da Silva, Lide- rança, Marabá-PA, 17/11/2005 / José dos Reis Conceição, Trab. Rural, Marabá-PA, 1/3/2005 / Daniel Soares da Costa Filho, Sindicalista, Parauapebas-PA, 15/2/2005 / Antônio Matos Filho, Sindicalista, Parauapebas, 8/6/2005/ Carlos Coelho de Freitas, Sem-Terra, Parauapebas/Marabá-PA, 26/1/2005 / José Ribeiro do Nascimento, Sem-terra, Parauape- bas/Marabá-PA, 15/6/2005 / Raimundo Moraes Pinheiro, Liderança, Santarém-PA 20/5/2005 / Jailton da Silva Lago, Trab. Rural, São Félix do Xingu/Altamira-PA, 18/2/2005 / Francinalva Conceição Silva,

\section{7}




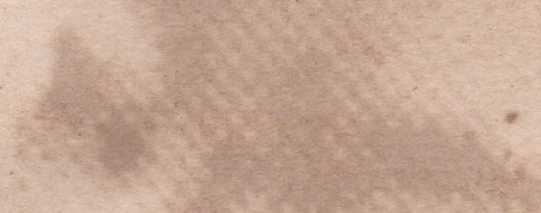

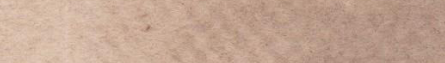

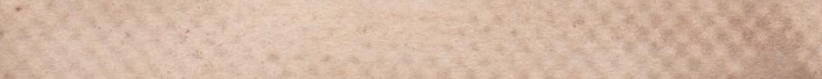

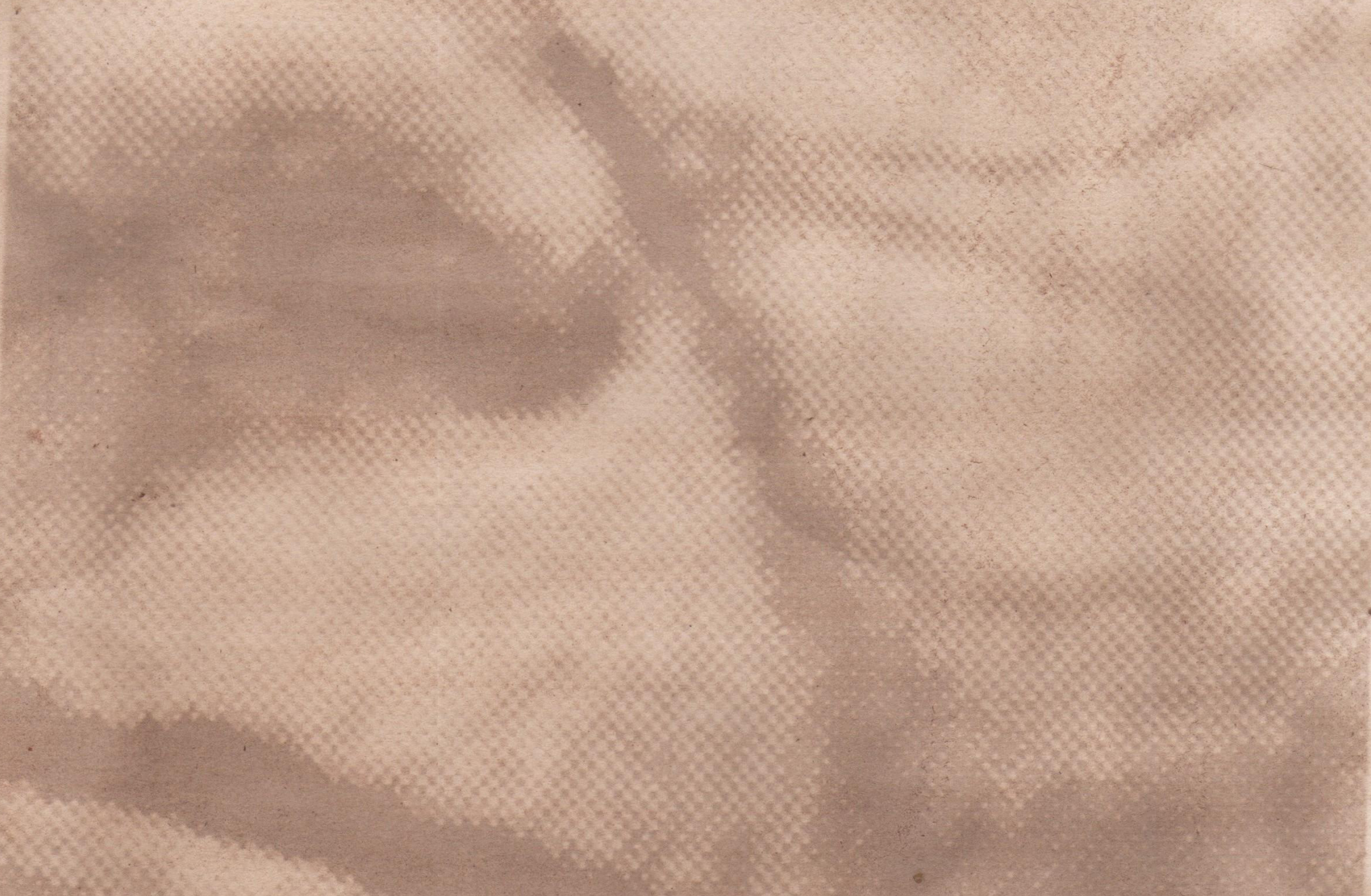



Sem-terra, Tucuruí-PA, 8/ 1/2005 / José Freitas de Melo, Posseiro, arecis-RO, 15/5/2005 / José Santos, "Pimenta", Ribeirinho, Barcelos-AM, 14/11/2006 / Gedeão Rodrigues da Silva, Dirigente Sindical, Lábrea-AM, 27/2/2006 / Cláudio Souza de Oliveira, Posseiro,Colniza-MT,28/8/ 2006 / José Roberto Tranqüilo, Posseiro, Colniza, 28/8/2006 / Trabalhador da Fazenda do Jaime 1, Trab. Rural, Altamira-PA, 14/6/2006 / Trabalhador da Fazenda do Jaime 1, Trab. Rural, Altamira-PA, 14/6/2006/ Antônio Augusto, Trab. Rural, Anapú/ Pacajá-PA, 10/10/2006 / Izaura Alves Muniz, Sem-terra,Baião-PA, 20/6/ 2006 / Tiago Alves Muniz, Sem-terra, Baião-PA, 20/6/2006 / Tatiane Alves Muniz, Sem-terra, Baião-PA, 20/6/2006 / Raimun- do Nonato Muniz, Sem-terra, Baião-PA, 20/6/2006 / Domingos Carneiro, Liderança, Bannach-PA, 8/1/2006 / Cícero Jacinto de Jesus, Sem-terra, Cumaru do Norte-PA, 4/9/2006 / Almir Pedro de Paula, Sem-terra, Cumarudo Norte-PA, 4/9/2006 / José Lopes do Carmo, Sem-terra, Itupiranga-PA, 
17/8/2006 / Genivaldo, Liderança, Marabá/Itupiranga-PA, 22/1/2006 / Antônio Barbosa da Silva, Sem-terra, Moju-PA, 31/5/2006 / Raimundo Nonato da Silva "Cinato", Lideran- ça, Moju-PA, 20/9/2006 / Antônio Carlos de Souza Bezerra, Trab. Rural, Ourilândia-PA, 16/1/2006 / Trab. Rural da Com. S. Miguel Arcanjo, Ribeirinho, Portel-PA, 20/7/2006 / Sr. Cabral, Sem-terra, São Domingos do Araguaia-PA, 20/7/2006 / Antônio Bezerra da Silva, Trab. Rural, São Félix do Xingu-PA, 8/1/2006 / Henrique Aparecido Ribeiro, Criança, São Félix do Xingu -PA, 4/6/2006 / Gerson Cristo, Político, São Félix do Xingu-PA, 7/10/2006 / Francisco Cleves Guimarães de Araújo, Liderança, Tucuruí-PA, 10/7/2006 / Jorge das Mercedes Roque, Liderança, Tucuruí-PA, 3/12/2006 / Manoel Coelho dos Santos, Liderança, Tucuruí/Itupiranga-PA, 5/7/2006 / Valdeir Joaquim Santiago, Assen- tado, Xinguara-PA, 17/12/2006 / José Vanderlei Rarvewfki, "Polaco", Sem-terra, Campo Novo de Rondônia-RO, 26/3/2006 / Nélio Lima Azevedo, "Pindaíba", Sem-terra, Campo Novo de Rondônia-RO, 30 26/3/2006 / Maurício, "Caubói”, Sem Informação, Lábrea-AM, 30/1/2007 / Gênesis Félix, Liderança, Lábrea-AM, 13/03/2007 / Tomé Gua- jajara, Índio, Buritirana/ Buriticupu/Bom Jesus das Selvas/ Arame/ Amarante do Maranhão T.I. Araribóia-MA, 15/10/2007 / Alfredo Pereira, Pequeno Proprietário, Codó,-MA, 28/8/2007 / João Carlos Pontes, Assentado, Altamira-PA, 22/01/2007 / Marcos José Moraes Pereira, Pequeno proprietário, Conceição do Araguaia-PA, 10/05/2007 / Antônio Santos do Carmo, Sem-terra, Irituia-PA, 02/05/2007 / Antônio Raimundo dos Santos, "Carequinha", Liderança, Novo Repartimento-PA, 26/04/2007 / Manoel da Conceição Cruz Filho"Manoel Borracheiro", Liderança, Rondon do Pará/ Dom Eliseu-PA, 23/10/2007 / "Camarão", Sem-terra, Nova Mamoré-RO, 23/3/2007 / Edmilson, Trab. Rural, Araguaína/ Santa Fé do Araguaia-TO, 19/7/2007 / José Reis, "Zé Preto", Trab. Rural, Araguantins-TO, 04/04/2007 / Francisco da Silva, Liderança, Lábrea-AM, 22/02/2008 / Maria dos Anjos Guajajara, Índio, Buritirana/Buriticupu/Bom Jesus das Selvas/Arame/ Amarante doMaranhãoT.I. Araribóia-MA, 05/05/2008 / Hélio Luiz Gonzaga, Trab. Rural, Confresa/Vila Rica/Santa Terezinha-MT, 04/03/2008/Josué, Sem-terra, Goianésia-PA, 20/02/2008 / Jovelino Alves Leite [Neno], Trab. Rural, Novo Repartimento/Baião-PA, 26/04/2008 / José Francisco Alves Leite, Trab. Rural, Novo Repartimento/ Baião-PA, 26/04/2008 / Josimar Elízio Barbosa, Sindicalista, Parauapebas/Serra Pelada-PA, 07/05/2008/ Manoel Ba- tista de Oliveira, Garimpeiro, Parauapebas/Serra Pelada-PA, 22/11/2008 / José Ribamar Rodrigues dos Santos, Sem-ter-ra, Redenção-PA, 12/11/2008 / [nome não informado], Trab. Rural, São Félix do Xingu-PA, 18/10/2008 / Alderico Mário de Brito, Sindicalista, São João do Araguaia-PA, 24/10/2008 / Pai e filho [nomes não informados], Trab. Rurais, Tailândia-PA, 
30/05/2008 / Raimundo Agnaldo Dourado de Almeida, Liderança, Tucuruí-PA, 27/09/2008 / Emival Bar- bosa Machado, Assentado, Tucuruí-PA, 24/04/2008 / Diassis Lima, Sem-terra, Xinguara/ Conceição do Araguaia-PA, 31/01/2008 / Edson Dutra, Aliado, Campo Novo de Rondônia-RO, 29/04/2008 / Evandro Dutra Pinto, Sem-terra, Porto Velho-RO, 20/11/2008 / Edmilson Gomes de Oliveira, Sem-terra, Porto Velho-RO, 20/11/2008/ Adalto da Silva FiIho, Sem-terra, Porto Velho-RO, 20/11/2008/ Francisco, "Mineiro", Posseiro, Plácido de Castro-AC, 09/01/2009 / Agenor de Sousa Pereira, Quilombola, São Luís Gonzaga do Maranhão-MA, 11/07/2009/ Edelton Rodrigues do $\mathrm{N}$ a s c imento, Sem-terra, Bom jesus do Araguaia/Serra Nova Dourad a - M T,

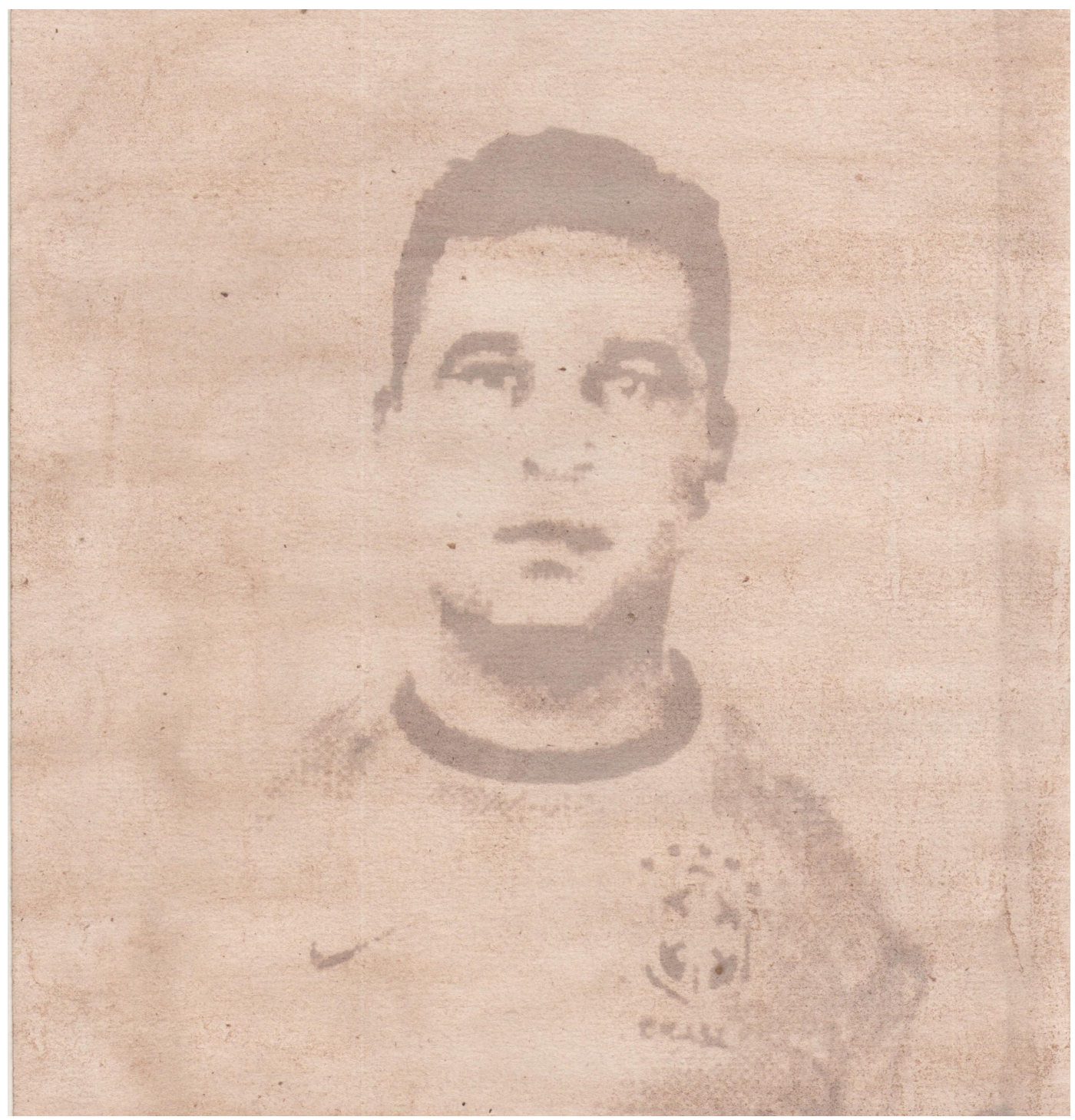


17/06/2009 / Abiner José da Costa, Sem-terra, Bom Jesus do Araguaia/Serra Nova Dourada-MT, 17/06/2009 / Ademar Oliveira e Silva, Liderança, Colniza-MT, 08/08/2009/ Manoel Francisco Silva Souza, Liderança, Breu Branco-PA, 01/02/2009 / Joel Silva do Nascimento, Assentado, Breu Branco-PA, 18/10/2009 / Saturnino Pereira Silva, Aliado, Dom Eliseu-PA, 29/09/2009 / Wagner Nascimento da Silva, Sem-terra, Marabá-PA, 23/08/2009 / Edvan de Amarantes, Sem-terra, Rondon do Pará-PA, 24/04/2009 / Luiz Lopes Barros, Liderança, Tailândia-PA, 12/06/2009 / Adair da Silva Almeida, Liderança, Tailândia-PA, 20/10/2009 / Altiro, Sem-terra, Tucumã-PA, 05/10/2009 / Élcio Machado, "Sabiá", Liderança, Buritis-RO, 09/12/2009 / Yekuana Luiz Vi- cente Carton, indígena, Mucajaí/ Alto Alegre/CaracaraîT. I.Yanomami/Apiauí/Papiu/Yawaripé-RR, 21/01/2009 / Valmir de Souza, Liderança, Humaitá-AM, 03/01/2010 / Raimundo Pereira da Silva,"Raimundo Chagas", Liderança, Codó-MA, 14/01/2010 / Francisco Ribeiro Viana, Liderança, Liderança, Santa Luzia-MA, 20/4/2010 / Manoel Martins da Costa, Sem-terra, Eldorado dos Carajás-PA, 30/11/2010 / José Valmeristo Soares, "Caribé", Sem-terra, Irituia/ Santa Luzia do Pará-PA, 3/9/2010 / Raimundo Nonato, Liderança, Novo Repartimento Acamp. às margens do Lago daUHE de Tucuruí-PA, 21/1/2010 / Antônio Moreira da Cruz, "Chapéu de Couro", Assentado, Pacajá-PA, 17/9/2010 / Francisca Pereira da Cruz, Assentada, Pacajá-PA, 17/9/2010 / Assentados do P.A. Cururuí de nome não identificado, Pacajá-PA, 18/9/2010 / José Feitosa da Silva, Assentado, Pacajá-PA, 18/9/2010 / Cícero Santiago dos Santos,"Índio", Liderança, Pacajá-PA, 18/9/2010 / Pedro Alcantâra de Souza, Liderança, Redenção-PA, 31/3/2010 / Paulo Roberto Paim, Liderança, Santana do Araguaia-PA, 28/5/2010 / José Jacinto Gomes, "ZéPretinho", Sem-terra, Santana do Araguaia-PA, 26/6/2010 / Givaldo Vieira Lopes, Sem-terra, Santana do Araguaia-PA, 22/10/2010 / Lourival Coimbra Gomes,"Baia- no", Sem-terra, Santana do Araguaia-PA, 24/10/2010 / Baltazar, Trab. Rural, São Félix do Xingu-PA, 13/10/2010 / Valmir da Silva, Trab. Rural, São Félix do Xingu-PA, 16/8/2010 / Francisco Ferreira da Silva, Pequeno proprietário, Porto Velho-RO, 27/02/2010 / Gabriel Vicente de Souza Filho, Sem-terra, Palmeirante-TO, 16/10/2010 / João Doido, Posseiro, Sena Madureira-AC, 3/11/2011 / Adelino Ramos, "Dinho", Liderança, Lábrea-AM, 27/5/2011 / Criança Awá-Guajá, Indígena, ArameT. I. Arariboia/92 Aldeias/ EtniasGuajajara, Gavião e Guajáĺndio-MA, 30/10/2011 / Júlio Luna da Silva, Assentado, Arame-MA, 08/12/2011 / Tazirã Ka'apor, Indígena, Centro do Guilherme T. I. Alto Turiaçu-MA, 031/3/2011 / João Conceição da Silva, Assentado, Santa Luzia-MA, 24/9/2011 / Cícero Felipe da Silva, "Cícero", Lide- rança, Santa Luzia-MA, 6/2/2011 / Francisco Alves Macedo, Liderança, Breu Branco-PA, 3/3/2011 / João Chupel Pri- mo, 
Ag. Pastoral, Itaituba-PA, 22/10/2011 / Pedro Oliveira Teixeira, "PedroSacaca", Sindicalista, Itupiranga-PA, 29/1/2011 / Jurandir Soares Nunes, Assentado, Juruti-PA, 12/1/2011 / Valdemar Oliveira Barbosa,"Piauí", Liderança, Marabá-PA, 25/8/2011/ Maria do Espírito Santo da Silva, Liderança, Nova Ipixuna-PA, 24/5/2011/ José Cláudio Ribei- ro da Silva, Liderança, Nova Ipixuna-PA, 24/5/2011 / Herenilton Pereira dos Santos, Assentado, Nova Ipixuna-PA, 26/5/2011 / Nildo Ferreira, Assentado, Pacajá-PA, 30/4/2011 / Adão Ribeiro, Assentado, Pacajá-PA, 30/4/2011 / Obede Loyla Souza, Sem-terra, Pacajá-PA, 19/6/2011 / José Ribamar Teixeira dos Santos, "Riba", Liderança, Rondon do Pará-PA, 7/10/2011 / Wdiley Alves Martins, Pequeno Proprietário, Porto Velho-RO, 12/3/2011 / Suelen Cristina Rodrigues Ferreira, Pequena Proprietária, Porto Velho-RO, 12/3/2011 / Francisnilson João Constante de Souza, Pequeno Proprie- tário, Apuí-AM, 15/7/2012 / Raimundo Nonato da Silva Chalub, "Rato Branco", Liderança, Lábrea-AM, 21/11/2012/Raimundo Alves Borges," Cabeça”, Liderança, Buriticupu-MA, 14/4/2012 / Francisco da Conceição Souza Guajajara, Indígena, Grajaú Bacurizinho/Aldeia Kamihaw Guajajara/Nazaré-MA, 09/03/2012 / Maria Amélia Guajajara, Indígena, GrajaúT. I. Canabrava/Aldeias Coquinho/Coquinho II/Iha de São Pedro-MA, 28/04/2012/Adenilson Kirixi Mun- duruku, Indígena, Alta Floresta Aldeia Teles Pires/EtniasMunduruku e Kayabi-MT, 7/11/2012 / Abiair Amaral Gusmão, Quilombola, Acará-PA 07/11/2012 / Josivani Amaral Gusmão, Quilombola, Acará-PA, 7/11/2012 / Mamede Gomes de Oliveira, Liderança, Belém-PA, 23/12/2012 / Edvaldo da Silva, "Divaldinho", Assentado, Itaituba-PA, 16/01/2012 / Lelo Akay Munduruku, Indígena, Jacareacanga . I. Munduruku/ UHETapajós-PA, 23/06/2012 / Célio Tigre, Assentado, Novo Progresso-PA, 15/08/2012 / Renato Nathan Gonçalves Pereira, Liderança, Ariquemes-RO, 10/4/2012 / Gilberto Tiago Brandão, Liderança, Machadinho d'Oeste, 25/02/2012 / Ercias Martins de Paula, Liderança, Machadinho d 'Oes- te-RO, 01/03/2012/Orlando Pereira Sales, "Paraíba", Liderança, Nova Brazilândia do Oeste-RO, 29/11/2012 / João Oliveira da Silva Kaxarari, Indígena, Porto Velho-RO, 26/08/2012/Dinhana Nink, Ocupante, Porto Velho-RO, 30/03/2012 / José Barbosa da Silva, "ZéAlbino", Pequeno Proprietário, Seringueiras-RO, 15/05/2012 / José Carlos Al- ves de Almeida, Ocupante, Vilhena-RO, 31/12/2012/ Fabiana Pereira de Souza, Ocupante, Vilhena-RO, 31/12/2012 / Margarido, Sem-terra, Bujari-AC, 13/12/2013 / Ivan Tenharim, Indígena, Humaitá-AM, 2/12/2013 / Gilmar dos Santos de Jesus, Assentado, Bom Jesus das Selvas-MA, 20/02/2013 / Rosivaldo Mesquita Sousa, Posseiro, Icatu-MA, 28/9/2013/Machado, Assentado, Satubinha-MA, 26/4/2013/Reginaldo Alves Pereira, Assentado, Confresa-MT, 25/10/2013 / Josimar Lima Silva, Sem - terra, Novo Mundo-MT, 16/4/2013 / Antônio 
Ferreira Caetano,"Toinzinho", Pequeno pro- prietário, Vila Rica-MT, 19/7/2013 / Gilzan Teixeira Lima, Liderança, Altamira-PA, 16/03/2013 / Washington de Freitas Martins, Trab. Rural, Goianésia-PA, 9/9/2013 / Antônio Maciel, Sem-terra, Itupiranga-PA, 30/11/2013 / Wilson Ambró- sio da Silva, Indígena, Itupiranga Aldeia Ororubá/Reserva dos Atikum-PA, 39/7/2013 / Trabalhador da Faz.Catuxo, Trab. Rural, Parauapebas-PA, 19/4/2013 / Welbert Cabral Costa, Trab. Rural, São Félix do Xingu-PA, 24/7/2013 / Pau- lo César Cordoval Ferreira, Posseiro, Vilhena-RO, 9/5/2013 / Índios Yanomami, Alto AlegreT. I.Yanomami/Apiauí/ Papiu/Yawaripé-RR, 14/4/2013 / Criança Indígena Yanomami, Alto AlegreT. I.Yanomami/Apiauí/Papiu/Yawaripé-RR, 14/4/2013 / Luís Carlos Silva, Liderança, Presidente Vargas-MA, 5/1/2014 / Josias Paulino de Castro, Liderança, Colni- za-MT, 16/8/2014 / Ireni da Silva Castro, Liderança, Colniza-MT, 16/08/2014 / Artêmio Gusmão, Quilombola, Acará-PA, 4/7/2014 / Aguinaldo Ribeiro Queiroz, Sem-terra, Bom Jesus do Tocantins-PA, 17/09/2014 / Jair Cleber dos Santos, Sem-terra, Bom Jesus do Tocantins-PA, 17/09/2014 / Leônidas Fernandes da Silva, Sem-terra, Brejo Grande do Ara- guaia-PA, 01/06/2014 / Maria Paciência dos Santos, Sem-Terra, Eldorado dos Carajás-PA, 12/08/2014 / Lelis Lima Silva, Sem informação, Rio Maria-PA, 02/12/2014 / Aldinei Lima Silva, Sem Informação, Rio Maria-PA, 02/12/2014 / Welliomar Jackson da Silva, Posseiro, Santana do Araguaia-PA, 17/12/2014 / Félix Leite dos Santos, Liderança, São Félix do Xingu-PA, 18/07/2014 / Lúcio Gonçalves dos Santos, Sem-terra, Alto Alegre do Parecis-RO, 18/12/2014 / Rodrigo Pereira de Freitas, Sem-terra, Porto Velho-RO, 09/07/2014 / Acampado na Gleba Rio das Garças, Sem-terra, Porto Velho-RO, 17/07/2014 / Rubens Santiago do Nascimento, Sem-terra, Porto Velho-RO, 24/04/2014 / Odair Ruiz, Assentado, Vilhena-RO, 04/09/2014 / Maria das Dores dos Santos S.Priante, "Dora", Liderança, Iranduba-AM, 12/8/2015 / Raimundo Pires Ferreira, Posseiro, Amarante-MA, 1/6/2015 / Zilquenia Machado Queiroz, Posseiro, Ama- rante-MA, 30/06/2015 / Raimundo Santos Rodrigues, Ambientalista, Bom Jardim-MA,25/8/2015/Eusébio Ka'apor, Indígena, Centro do Guilherme T. I. Alto Turiaçu-MA, 26/04/2015 / Antônio Isídio Pereira da Silva, Liderança, Codó-MA, 24/12/2015/Wislen Gonçalves Barbosa, Funcionário Público, Anapu-PA,17/11/2015 / Hércules Santos de Souza, Sem-terra, Anapu-PA, 10/10/2015/ Edinaldo Alves Moreira,"Lourinho", Sem-terra, Anapu-PA, 05/07/2015/ Jesusmar Batista Farias, "Suis", Sem-terra, Anapu-PA, 11/08/2015/Cosmo Pereira de Castro, Sem-terra, Anapu-PA, 22/08/2015/ José Nunes da Cruz, "Zé da Lapada", Sem-terra, Anapu-PA, 27/10/2015 / Cláudio Bezerra da Costa,"Ivanzinho", Sem-terra, Anapu-PA, 31/10/2015/Wesley Washington Sousa Barros, Sem-terra, Conceição do Araguaia-PA, 17/02/2015/Samylla Letícia Souza Muniz, Sem-terra, Conceição do Araguaia-PA, 17/02/2015 / Matheus Sousa Barros, Sem-ter- 

ra, Conceição do Araguaia-PA, 17/02/2015 / Júlio César Souza Muniz, Sem-terra, Conceição do Araguaia-PA, 17/02/2015/Leidiane Souza Soares, Sem-terra, Conceição do Araguaia-PA, 17/02/2015/Washington Miranda Muniz, Sem-terra, Conceição do Araguaia-PA, 17/02/2015 / Marcus, Trab. Rural, Floresta do Araguaia-PA, 14/01/2015 / João Miranda, Sem-terra, São Félix do Xingu-PA, 15/05/2015 / Osvaldo Rodrigues Costa, Sem-terra, São Félix do Xingu-PA, 06/11/2015 / José Osvaldo Rodrigues de Sousa, Sem-terra, Tucuruí-PA, 14/06/2015 / Daniel Vilanova Dias, Atingido por barragem, Vitória do XinguSítio Pimental/UHE de BeloMonte-PA, 18/05/2015 / Leidiane Drosdroski Machado, Atingida por barragem, Vitória do XinguSítio Pimental/UHE de BeloMonte-PA, 18/05/2015 / José Antônio Dória dos Santos,"Zé Minhenga", Sem-terra, Alto Paraíso-RO, 27/1/2015 / Trabalhadores Sem-Terra/Faz.Formosa, Alto Paraíso-RO, 11/05/2015 / Francimar de Souza, Sem-terra, Alto Paraíso-RO, 11/12/2015/Odilon Barbosa do Nascimento, Posseiro, Buritis-RO, 10/04/2015 / Jander Borges Farias, Liderança, Buritis-RO, 17/04/2015 / Paulo Justino Pereira, Liderança, Buritis-RO, 01/05/2015/Delson Mota, "Capixaba", Liderança, Buritis-RO, 15/07/2015 / Altamiro Lopes Ferreira, Sem-terra, Costa Marques-RO, 04/03/2015 / Cloves de Souza Palma, Liderança, Cujubim-RO, 30/06/2015 / Fábio Carlos da Silva Teixeira, Sem-terra, Machadinho d'Oeste-RO, 12/04/2015/Trabalhador/Faz. Padre Cícero, Trab. Rural, Monte Negro-RO, 06/07/2015/Terezinha Nunes Meciano, Liderança, Monte Negro-RO, 22/11/2015 / Anderson Mateus André dos Santos, "Índio", Liderança, Monte Negro-RO, 22/11/2015 / Lucas da Costa Silva, Sem-terra, Monte Negro-RO, 31/12/2015 / Dagner Lemes Pereira, Trab. Rural, Vilhena-RO, 17/10/2015 / Daniel Aciari, "Ceará", Pequeno Proprietário, Vilhena-RO, 17/10/2015 / José Bezerra dos Santos, Trab. Rural, Vilhena-RO, 17/10/2015 / João Fernandes da Silva, Trab. Rural, Vilhena-RO, 17/10/2015 / João Pereira Sobrinho, Trab. Rural, Vilhena-RO, 17/10/2015 / Altamiro Ferreira Pinto, Posseiro, Presidente Figueiredo-AM, 11/05/2016 / Josué Gomes Pinto, Posseiro, Presidente Figueiredo-AM, 11/05/2016 / Aponuyre Guajajara, Indígena, Amarante do Maranhão [T. I. Arariboia]-MA, 26/03/2016 / Genésio Guajajara, Indígena, Amarante do Maranhão T. I. Arariboia-MA, 11/04/2016 / Isaías Guajajara, Indígena, Amarante do Maranhão T. I. Arariboia-MA, 19/04/2016 / Assis Guajajara, Liderança Indígena, Amarante do Maranhão T. I. Arariboia-MA, 22/04/2016 / Candide Zaraky Tenetehar, Indígena, Amarante do Maranhão T. I. Arariboia-MA, 28/07/2016 / Roni dos Santos Miranda, Sindicalista, Amarante do Maranhão-MA, 01/02/2016/José Queirós Guajajara, Liderança Indíge- na, Grajaú T. I. Cana Brava-Marajá-MA, 23/09/2016/Cacique José Colírio Oliveira Guajajara, Liderança Indígena, Grajaú, T. I. Cana Brava-Marajá-MA, 26/11/2016/ José Dias 
de Oliveira Lopes Guajajara, Liderança Indígena, Grajaú, T. I. Bacurizinho-MA, 12/11/2016 / Marrone, Sem-terra, Anapú-PA, 08/06/2016 / Titela, Sem-terra, Anapú-PA, 08/06/2016 / João Luiz de Maria Pereira, Funcionário Público, Novo Progresso-PA, 17/06/2016 / Adoaldo Rodrigues Barbosa, Sem-terra, Santana do Araguaia-PA, 20/09/2016 / Luiz Antônio Bonfim, Liderança, São Domingos do Ara- guaia-PA, 12/02/2016 / Ronair José de Lima, Liderança, São Félix do Xingu-PA, 04/08/2016 / Valdomiro Lopes de Lorena, Sem-terra, Nova Bandeirantes-MT, 16/06/2016 / Edilene Mateus Porto, "Edilena", Liderança, Alto Paraíso-RO, 13/09/2016 / Isaque Dias Ferreira, Liderança, Alto Paraíso-RO, 13/09/2016 / Vanderlei Domingues Rodrigues,"Nem", Sem-terra, Alto Paraíso-RO, 25/09/2016 / Milton Rodrigues, Sem-terra, Alto Paraíso-RO, 11/10/2016 / Antônio Bento Cardoso Júnior, “Toizinho”, Sem-terra, Alto Paraíso-RO, 11/10/2016 / Jesser Batista Cordeiro, Sem-terra, Buritis-RO, 24/04/2016 / Nivaldo Batista Cordeiro, Sem-terra, Buritis-RO, 24/04/2016 / José Cândido Lopes Filho, "ZéBarba", Pequeno Proprietário, Buritis-RO, 02/09/2016/Geraldo de Campos Bandeira, Sem-terra, Buritis-RO, 09/05/2016 / Cleidiane Alves Teodoro, Sem-terra, Buritis-RO, 22/05/2016 / Luís Carlos da Silva, Liderança, Buritis-RO, 22/05/2016/ Ruan Hildebran Aguiar, Sem-terra, Cujubim-RO, 31/01/2016 / Alysson Henrique Lopes, Sem-terra, Cujubim-RO, 31/01/2016 / Adna Senhora Teixeira, Liderança, Cujubim-RO, 06/07/2016 / Cleverson Carneiro, Trab. Rural, Espigão do Oeste-RO, 26/05/2016 / Enilson Ribeiro dos Santos, Liderança, Jaru-RO, 23/01/2016 / Valdiro Chagas de Moura, Liderança, Jaru-RO, 23/01/2016 / Avildes Alves Pereira, Posseiro, Machadinho d'Oeste-RO, 19/10/2016 / Luciano Fer- reira de Andrade, Liderança, Mirante da Serra-RO, 18/08/2016 / Nilce de Souza Magalhães,"Nicinha", Liderança, Por- to Velho, 07/01/2016 / Sebastião Pereira dos Santos, Sem-terra, Vale do Paraíso-RO, 28/09/2016 / Geraldo Lucas, Sem-terra, Bonfim-RR, 17/12/2016 / Genivaldo Braz do Nascimento, "Ninja", Liderança, Araguaína-TO, 08/07/2016 / Casimiro Batista de Oliveira, Assentado, Porto Nacional-TO, 26/06/2016 / Luís Jorge de Araújo, Liderança, Wanderlân- dia-TO, 05/08/2016 / Flávio de Lima de Souza, Liderança, Canutama-AM, 14/12/2017 / Marinalva Silva de Souza, Li- derança, Canutama-AM, 14/12/2017 / Jairo Feitosa Pereira, Liderança, Canutama-AM, 14/12/2017 / José Caneta Ga- vião, "Cu Carut", Indígena, Amarante do Maranhão-MA, 06/03/2017 / Sônia Vicente Cacau Gavião, "CryCapric", Indígena, Amarante do Maranhão-MA, 06/03/2017 / Valmir Rangeu do Nascimento, Posseiro, Colniza-MT, 19/04/2017/ Fábio Rodrigues dos Santos, Posseiro, Colniza-MT, 19/04/2017 / Francisco Chaves da Silva, Posseiro, Colniza-MT, 19/04/2017 / Samuel Antônio da Cunha, Posseiro, Colniza-MT, 19/04/2017 / Aldo Aparecido Carlini, Possei- 
ro, Colni- za-MT, 19/04/2017 / Edison Alves Antunes, Posseiro, Colniza-MT, 19/04/2017 / Ezequias Santos de Oliveira, Posseiro, Colniza-MT, 19/04/2017 / Izaul Brito dos Santos, Posseiro, Colniza-MT, 19/04/2017/ Sebastião Ferreira de Souza, Posseiro, Colniza-MT, 19/04/2017 / Oseir Rodrigues da Silva, Sem-terra, Abaetetuba-PA, 24/05/2017 / Pereira Milho- mem, Sem-terra, Abaetetuba-PA, 24/05/2017 / Jhonatan Alves Pereira dos Santos, "Jhon", Sem-terra, Anapú-PA, 20/07/2017 / Fernando Pereira, Liderança, Barcarena-PA, 22/12/2017 / Kátia Martins de Souza, Liderança, Castanhal-PA, 04/05/2017 / Etevaldo Soares Costa, Sem-terra, Curionópolis-PA, 04/05/2017 / Maria da Lurdes Fernandes Silva, Assentada, Itupiranga-PA, 25/07/2017 / Manoel Índio Arruda, "Índio", Assentado, Itupiranga-PA, 25/07/2017 / Eraldo Moreira Luz, "Pirata", Liderança, Marabá-PA, 13/09/2017/ Jorge Matias da Silva, Sem-terra, Marabá-PA, 13/09/2017/ Maria Trindade da Silva Costa, Liderança Quilombola, Moju-PA, 23/06/2017/ Waldomiro Costa Pereira, Assentado, Parauapebas-PA, 20/03/2017 / Hércules Santos de Oliveira, Sem-terra, Pau D Arco-PA, 24/05/2017 / Regivaldo Pereira da Silva, Sem-terra, Pau D Arco-PA, 24/05/2017 / Bruno Henrique Pereira Gomes, Sem-terra, Pau D Arco-PA, 24/05/2017/ Weldson Pereira da Silva, Sem-terra, Pau D Arco-PA, 24/05/2017/ Weclebson Pereira Milhomem, Sem-terra, Pau D Arco-PA, 24/05/2017 / Nelson Souza Milhomem, Sem-terra, Pau D Arco-PA, 24/05/2017 / Jane Júlia de Oliveira, Lide- rança, Pau D Arco-PA, 24/05/2017 / Ronaldo Pereira de Souza, Sem-terra, Pau D Arco-PA, 24/05/2017 / Rosenilton Pereira de Almeida, "Negão", Liderança, Rio Maria-PA, 07/07/2017 / Antônio Alves Gomes, "Toninho”, Sem-terra, Santana do Araguaia-PA, 02/05/2017 / Vanderson Siqueira dos Santos, Sem-terra, Ariquemes-RO, 13/06/2017 / Rober- to Santos Araújo, Liderança, Ariquemes-RO, 01/02/2017 / Hugo Rabelo Leite, Liderança, Candeias do Jamari-RO, 07/12/2017 / Liversino Azevedo, "Vítor", Assentado, Corumbiara-RO, 05/06/2017 / Elivelton Castelo Nascimento, Li- derança, Cujubim-RO, 15/02/2017 / Orestes Rodrigues de Castro, Sem-terra, Cujubim-RO, 04/03/2017 / Renato Souza Benevides, "Baixinho", Sem-terra, Machadinho D Oeste-RO, 04/03/2017 / Jefferson Neponuceno, Sem-terra, Macha- dinho D Oeste-RO, 13/07/2017 / Weverton Cantão, Sem-terra, Machadinho D Oeste-RO, 10/02/2017 / Paulo Sérgio Bento Oliveira, Sem-terra, Mirante da Serra-RO, 16/05/2017 / Valdenir Juventino Izidoro, "Lobo", Liderança, Ouro Preto do Oeste-RO, 05/06/2017 / Manoel Quintino da Silva Kaxarari, Liderança Indígena, Porto Velho-RO, 26/06/2017/ Ademir Souza Pereira, Liderança, Porto Velho-RO, 06/07/2017 / Ceará, Sem-terra, Rio Crespo-RO, 29/01/2017 / Valdinei Assis da Silva, "Nei", Aliado, Vilhena-RO, 29/04/2017 / Yure Silva, Aliado, Vilhena-RO, 29/04/2017 / Geo- vane Alves de Jesus, Aliado, Vilhena-RO, 
29/04/2017 / Erivelton Tenharin, Liderança Indígena, Colniza-MT, 10/10/2018/ Nazildo dos Santos Brito, Liderança QuiIombola, Acará-PA, 14/04/2018 / Aluísio Sampaio dos Santos, "Alenquer", Liderança, Altamira-PA, 11/10/2018 / Gazimiro Sena Pacheco, "Gordinho", Sem-terra, Anapu-PA, 09/01/2018 / Valde- mir Resplandes dos Santos, "Muletinho", Liderança, Anapu-PA, 09/01/2018 / Leoci Resplandes de Sousa, Sem-terra, A n a p u - P A, 03/06/2018 / Paulo

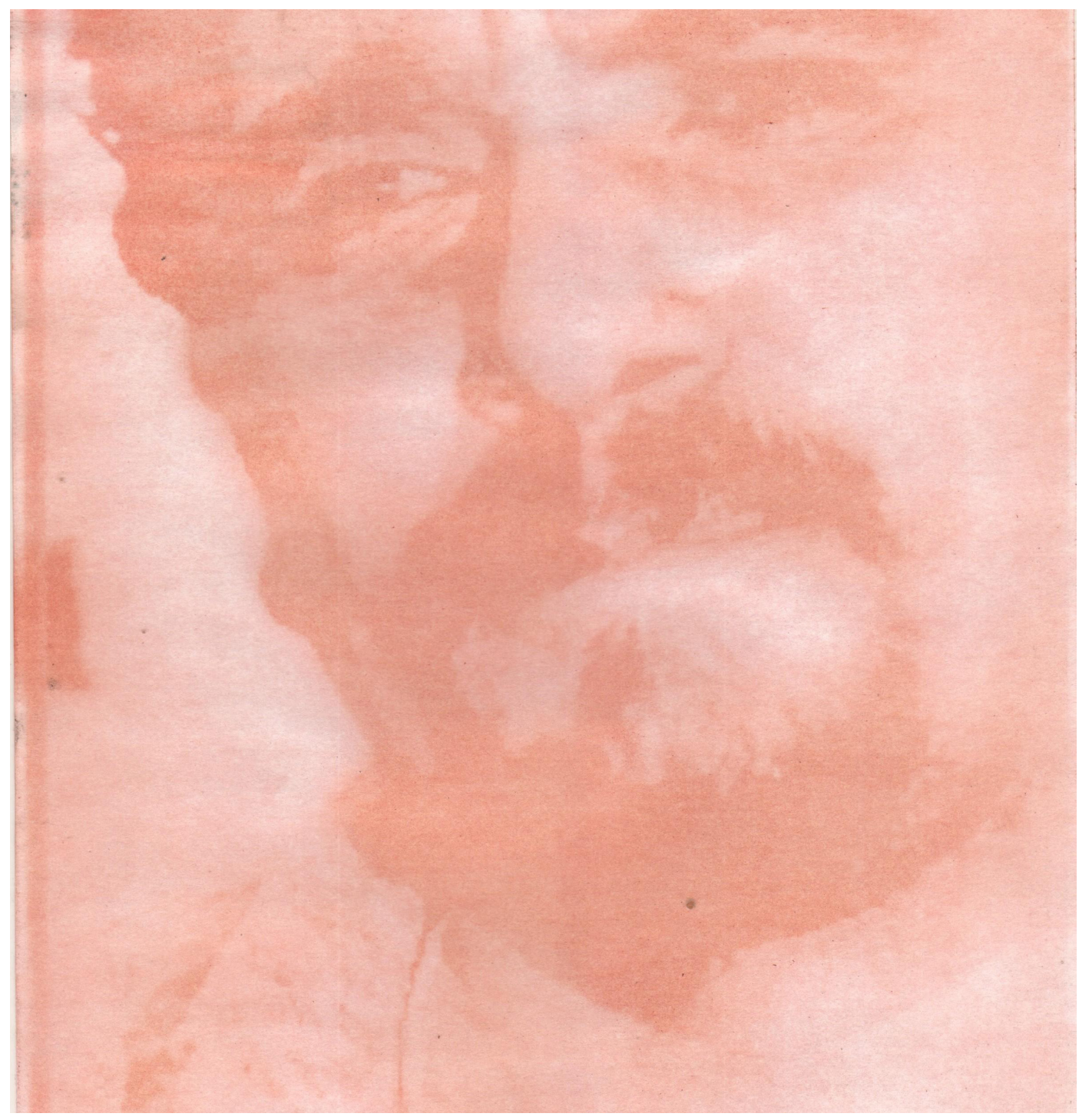
Sérgio Almeida Nas-

cimento, Liderança, Barcarena-PA, 12/03/2018 / Arleis Pereira de Sousa, Trab. Rural, Marabá-PA, 24/08/2018 / Paulo de Tasso Mendes da Silva, Trab. Rural, Marabá-PA, 24/08/2018 / Romar Roglin, Assentado, Novo 

Progresso-PA, 12/01/2018 / Antônio Rodrigues dos Santos, Assentado, Novo Progres- So-PA, 18/05/2018 / Ricardo Roglin, Assentado, Novo Progresso-PA, 13/07/2018 / Joacir Fran Alves da Mota, Sem-ter- ra, Pau D Arco-PA, 04/03/2018 / Gilson Maria Temponi, "Mineiro", Liderança, Placas-PA, 15/12/2018 / Katyson de Souza, Liderança, Santa Isabel-PA, 02/06/2018 / Juvenil Martins Rodrigues, "Foguinho", Liderança, Santa Maria das Barreiras-PA, 01/08/2018 / Haroldo Betcel, Quilombola, Santarém-PA, 29/09/2018 / Tiago Campin dos Santos, Possei- ro, Nova Mamoré-RO, 25/07/2018 / Ademar Ferreira, Posseiro, Nova Mamoré-RO, 25/07/2018/Joscione Nunes das Neves, Trab. Rural, Ouro Preto do Oeste-RO, 09/02/2018 / Edemar Rodrigues da Silva, "Galego ou Lalaco", Lideran- ça, Ouro Preto do Oeste-RO, 03/04/2018 / Ismauro Fátimo dos Santos, Liderança, Seringueiras-RO, 22/07/2018 / Lu- cas de Lima Batista, Sem-terra, Vilhena-RO, 08/07/2018 / Emyra Waiãpi, Liderança Indígena, Pedra Branca do Ama- pari-AP, 22/07/2019 / Nemis Machado de Oliveira, Liderança, Lábrea-AM, 30/03/2019 / Denis, Posseiro, Lábrea-AM, 02/11/2019 / Cacique Willames Machado Alencar, Liderança Indígena, Manaus-AM, 13/06/2019 / Carlos Alberto Oliveira de Souza, "Mackpak", Liderança Indígena, Manaus-AM, 06/08/20191 / Cacique Francisco de Souza Pereira, Liderança Indígena, Manaus-AM, 27/02/20191/ Maxciel Pereira dos Santos, Funcionário Público, Tabatinga-AM, 06/09/2019 / Paulo Paulino Guajajara, Liderança Indígena, Bom Jesus das Selvas-MA, 01/11/2019 / Cacique Firmino Prexede Guajajara, Liderança Indígena, Jenipapo dos Vieiras-MA, 07/12/2019 / Raimundo Benício Guajajara, Lideran- ça Indígena, Jenipapo dos Vieiras-MA, 07/12/20191/ Francisco Sales Costa de Sousa, Liderança, Zé Doca-MA 18/12/2019/Elizeu Queres de Jesus, Posseiro, Colniza-MT, 05/01/2019/Sr. Gilberto, Liderança, Cotriguaçu-MT, 01/08/2019/ Márcio Rodrigues dos Reis, Liderança, Anapu-PA, 04/12/2019/Paulo Anacleto, Aliado, Anapu-PA, 09/12/2019 / Marciano dos Santos Fosalusa, Liderança, Anapu-PA, 20/02/2019 / Raimundo de Jesus Ferreira, Trab. Rural, Baião-PA, 24/03/20191 / Marlete da Silva Oliveira, Trab. Rural, Baião-PA, 24/03/2019 / Venilson da Silva Santos, Trab. Rural, Baião-PA, 24/03/2019 / Dilma Ferreira Silva, Liderança, Baião-PA, 22/03/2019/ Claudionor Amaro Costa da Silva, Assentado, Baião-PA, 22/03/2019/ Milton Lopes, Assentado, Baião-PA, 22/03/2019/ José Araújo dos Santos, Sem-terra, Conceição do Araguaia-PA, 26/09/2019 / Alexandre Coelho Furtado Neto, Liderança, Ourilândia do Norte-PA, 19/10/2019 / Carlos Cabral Pereira, Sindicalista, Rio Maria-PA, 11/06/2019 / Gustavo José Simoura, Sem- terra, Corumbiara-RO, 11/01/2019 / Zezico Guajajara, Indígena, Arame-MA, 31/03/2020 / Kwaxipuhu Ka'apor, Indí- gena, Centro do Guilherme-MA, 03/08/2020 / Ari Uru-eu-wau-wau, Indígena, Jaru-RO, 18/04/2020 [...] 
ZÉ CLAÚDIO, EXTRATIVISTA, JENIPAPO SOBRE PAPEL, FOTOCÓPIA 2019; EXTRAÇÃO DE PIGMENTO DE URUCUM, FOTOGRAFIA DIGITAL, 2019; PAULINO GUAJAJARA, INDÍGENA, URUCUM SOBRE PAPEL, FOTOCÓPIA 2020; DOROTHY STANG RELIGIOSA, JENIPAPO SOBRE PAPEL, FOTOCÓPIA 2019; MÃO PIGMENTADA DE JENIPAPO, FOTOGRAFIA DIGITAL, ILHA DE CARATATEUA, 2019; NAZILDO, QUILOMBOLA, JENIPAPO SOBRE PAPEL, FOTOCÓPIA 2019; HAROLDO BETCEL, QUILOM- BOLA, JENIPAPO SOBRE PAPEL, FOTOCÓPIA 2020; KÁTIA MARTINS, ASSENTADA, JENIPAPO SOBRE PAPEL, FOTOCÓPIA 2019; DEZINHO, SINDICALISTA, URUCUM SOBRE PAPEL, FOTOCÓPIA 2020; EMYRA WAIÃPI, INDÍGENA, JENIPAPO SOBRE PAPEL, FOTOCÓPIA, 2020. 